\title{
On the Robustness of the Damped $V$-Cycle of the Wavelet Frequency Decomposition Multigrid Method
}

\author{
A. Rieder ${ }^{1,2}$ and X. Zhou ${ }^{1}$, Houston \\ Received March 24, 1994; revised May 9, 1994
}

\begin{abstract}
Zusammenfassung
On the Robustness of the Damped $V$-Cycle of the Wavelet Frequency Decomposition Multigrid Method. The damped $V$-cycle of the wavelet variation of the "Frequency decomposition multigrid method" of Hackbusch [Numer. Math. 56, pp. 229-245 (1989)] is considered. It is shown that the convergence speed under sufficient damping is not affected by the presence of anisotropy but still depends on the number of levels. Our analysis is based on properties of wavelet packets which are supplied and proved. Numerical approximations to the speed of convergence illustrate the theoretical results.
\end{abstract}

AMS Subject Classifications: $65 \mathrm{~F} 10,65 \mathrm{~N} 30$

Key words: Wavelets, wavelet packets, robust multilevel methods, $V$-cycle.

Zur Robustheit des gedämpften $V$-Zyklus bei der FDMGM mit Wavelets. Wir betrachten den gedämpften $V$-Zyklus für die Wavelet-Variante der "Frequenzzerlegungs-Multigridmethode" von Hackbusch [Numer. Math. 56, 229-245 (1989)]. Es wird gezeigt, daß die Konvergenzgeschwindigkeit bei hinreichender Dämpfung durch Anisotropie nicht beeinflußt wird, aber noch von der Anzahl des Niveaus abhängt. Unsere Analyse beruht auf Eigenschaften von Wavelet-Paketen, die formuliert und bewiesen werden. Numerische Schätzungen der Konvergenzgeschwindigkeit erläutern die theoretischen Ergebnisse.

\section{Introduction}

An iterative method for solving a linear system arising by the discretization of the anisotropic model problem

$$
\begin{gathered}
-\varepsilon \frac{\partial^{2}}{\partial x^{2}} u(x, y)-\frac{\partial^{2}}{\partial y^{2}} u(x, y)+u(x, y)=f(x, y) \text { in } \Omega=(0, s)^{2}, \\
u \text { periodic, }
\end{gathered}
$$

$0<\varepsilon \leq 1$, is said to be robust if its rate of convergence (i.e. spectral radius of the iteration matrix) is bounded smaller than 1 uniformly in $\varepsilon$ and in the discretization step-size.

${ }^{1}$ Supported partially by AFOSR under grant number $90-0334$ which was funded by DARPA.

${ }^{2}$ Partially supported by a Feodor Lynen-Fellowship of the Alexander von Humboldt-Foundation. 


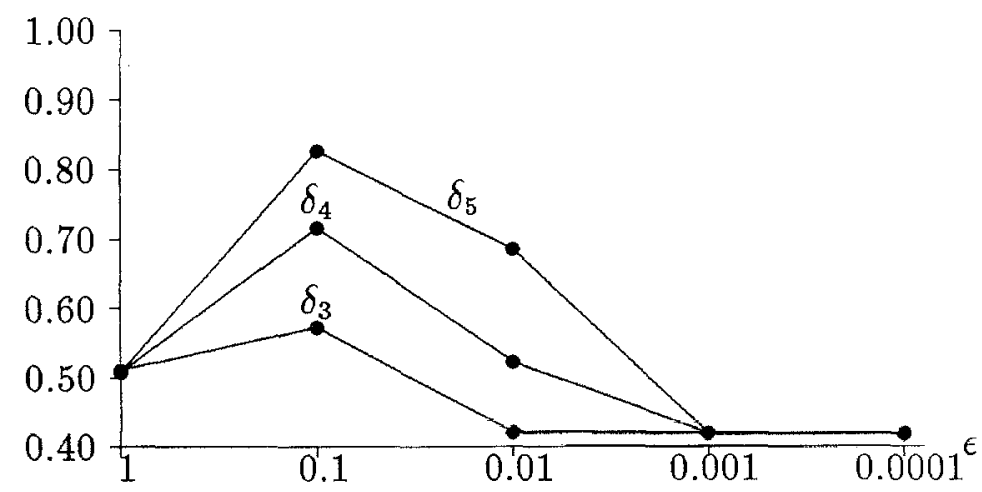

Figure 1. $V$-cycle convergence rates of the wavelet variation of the FDMGM for different discretization step-sizes: $\delta_{l}=2^{-l}$

A wavelet variation of the frequency decomposition mutigrid method (FDMGM) of Hackbusch [11] was presented in [16] and the robustness of the corresponding two-level method with respect to any intermediate level was verified.

However, in numerical experiments, the $V$-cycle variant of the multilevel method appeared not to be robust, see Fig. 1 (for a more detailed description of the experiment presented in Fig. 1 see Section 3.1). In this paper we are able to prove that a sufficiently damped version of the $V$-cycle is almost robust in the sense that the rate of convergence depends at most on the number of levels. Our result is of the same quality as typical estimates known for the convergence rates of multigrid solvers without regularity assumptions on the continuous problem, see [1], [3] and [17]. Indeed, the situation without regularity assumptions and the situation for the FDMGM are comparable in their lack of the approximation property. Hence, the standard proofs for the $V$-cycle convergence, see e.g. [10], are not applicable and adequate modifications are required which lead to level dependent convergence rates.

In the following section we shortly introduce the necessary vocabulary (wavelet packets, Mallat transformation, wavelet-Galerkin discretization) to understand the considerations in Section 3. Here, we prove the convergence of the damped $V$-cycle. Basically, we use the same techniques as in Chapter 7.2 of [10]. For the ease of presentation two auxillary results which are crucial but rather technical are given in the Appendix.

\section{Wavelet Analysis}

\subsection{Wavelet Packet System}

We will briefly recall various definitions and properties of wavelet packets [5] generated by the Daubechies scaling functions constructed in [6]. For a positive 
integer $N$, the Daubechies wavelet packets $\left\{\psi^{l}\right\}_{l \in \mathbf{N}_{0}}$ of order $N$ are defined as follows: There exist $2 N$ real numbers $a_{i}, i=0,1, \ldots, 2 N-1$, satisfying

$$
\sum_{k} a_{k}=2 \text { and } \sum_{k} a_{k+2 m} a_{k}=2 \delta_{0, m} \quad \text { for all } m \in \mathbf{Z},
$$

so that

$$
\begin{aligned}
\psi^{2 l}(x) & =\sum_{k=0}^{2 N-1} a_{k} \psi^{l}(2 x-k), \\
& l=0,1,2, \ldots \\
\psi^{2 l+1}(x) & =\sum_{k=0}^{2 N-1} b_{k} \psi^{l}(2 x-k),
\end{aligned}
$$

where $b_{k}=(-1)^{k} a_{2 N-k-1}$. The function $\psi^{0}$ is called scaling function and $\psi^{1}$ is called wavelet. All wavelet packets are compactly supported, with $\operatorname{supp}\left(\psi^{l}\right)=[0,2 N-1]$. For convenience, we define $a_{k}=0$ for $k \notin[0,2 N-1]$.

The wavelet packets are in $C^{\alpha(N)}$, the space of Hölder continuous functions with exponent $\alpha(N)$, where $\alpha(2) \approx .55, \alpha(3) \approx 1.09$ and $\alpha(N) \approx 0.2075 \cdot N$ for large $N$ [9]. We refer to [15] for comprehensive introduction to wavelet packets.

For the multilevel process defined in Section 3 we will need some of the second order connection coefficients ([2], [13])

$$
\Gamma_{k}^{l}:=\int_{\mathbf{R}}\left(\psi^{l}\right)^{\prime}(x-k)\left(\psi^{l}\right)^{\prime} d x, \quad 2-2 N \leq k \leq 2 N-2, \quad l \geq 0,
$$

of wavelet packets with $N \geq 3$. Due to the recursive definition of wavelet packets, their conection coefficients can be easily computed from the connection coefficients $\Gamma_{k}^{0}$ of the scaling function $\psi^{0}$.

\subsection{Mallat Transformations}

The (periodic) Mallat transformations $h, g: \mathbf{R}^{n} \rightarrow \mathbf{R}^{n / 2}, n$ even, of a vector $v \in \mathbf{R}^{n}$ are defined by

$$
\begin{aligned}
& (h v)_{k}=\frac{1}{\sqrt{2}} \sum_{l=0}^{2 N-1} a_{l} v_{l+2 k}, \\
& (g v)_{k}=\frac{1}{\sqrt{2}} \sum_{l=0}^{2 N-1} b_{l} v_{l+2 k},
\end{aligned}
$$

where we extend $v$ periodically, i.e. $v_{l}=v_{l+n}$. The coefficients $a_{l}$ in (2.5) and $b_{l}$ in (2.6) are those in (2.2) and (2.3), respectively. The Mallat transformations satisfy (see $[6],[14])$, 


$$
\begin{aligned}
& h^{t} h+g^{t} g=I, \\
& h h^{t}=g g^{t}=I, \\
& g h^{t}=h g^{t}=0 .
\end{aligned}
$$

We use I to denote the identity matrix of appropriate size throughout this paper.

\subsection{Wavelet-Galerkin Discretization of the Model Problem}

We introduce the Sobolev space $H_{p}^{1}(\Omega), \Omega=(0, s)^{2}$, with periodic boundary conditions,

$$
H_{p}^{1}=H_{p}^{1}(\Omega):=\left\{v \in L^{2}(\Omega): v_{x}, v_{y} \in L^{2}(\Omega), v(0, y)=v(s, y), v(x, 0)=v(x, s)\right\} .
$$

The weak or variational formulation of the model problem (1.1), (1.2), reads:

$$
\text { find } u \in H_{p}^{1}: \mathscr{A}(u, v)=\int_{\Omega} f v d x d y, \text { for all } v \in H_{p}^{1},
$$

where $\mathscr{A}$ is the $H_{p}^{1}$-elliptic bilinear form

$$
\mathscr{A}(u, v)=\int_{\Omega}\left(\varepsilon u_{x} v_{x}+u_{y} v_{y}+u v\right) d x d y
$$

Due to the Lax-Milgram theorem [4] (2.7) has a unique solution $u$. For a waveletGalerkin discretization of (2.7), we assume that $N \geq 3$ and that $s$ in (1.1) is an integer greater than $4 N-3$. Further, we set $\psi_{l, k}^{0}(x)=2^{l / 2} \psi^{0}\left(2^{l} x-k\right)$ and introduce the wavelet-Galerkin spaces

$$
V_{l}=V_{l}(0, s):=\left\{v \in L^{2}(0, s): v(x)=\sum_{k \in \mathbf{Z}} c_{k} \psi_{l, k}^{0}(x), x \in[0, s] \text {, and } c_{k}=c_{k+2^{l_{s}}}\right\} .
$$

Obviously, $V_{l}$ has the dimension $n_{l}=2^{l}$ s. The wavelet-Galerkin approximation $u_{l}$ to $u$ in the tensor product space $V_{l} \otimes V_{l} \subset H_{p}^{1}$ is the unique solution of

$$
\mathscr{A}\left(u_{l}, v_{l}\right)=\int_{\Omega} f v_{l} d x d y, \quad \text { for all } v_{l} \in V_{l} \otimes V_{l}
$$

A convergence proof of $u_{l} \rightarrow u$ is given in [18]. For $u_{l}$ we have the following expansion $u_{l}(x, y)=\sum_{i, j} u_{i, j}^{l} \psi_{l, i}^{0}(x) \psi_{l, j}^{0}(y)$ where the expansion coefficients $u_{i, j}^{l}$ are periodic with period $n_{l}$ in each index. We now define $f_{i, j}^{l}=\int_{\Omega} f(x, y) \psi_{l, i}^{0}(x) \psi_{l, j}^{0}(y) d x d y$. If we order the $u_{i, j}^{l}$ 's and $f_{i, j}^{l},{ }^{\prime}, 0 \leq i, j \leq n_{l}-1$, lexicographically and denote the resulting vectors $U_{l}$ and $F_{l}$, respectively, then we have the following linear system for the $n_{l}^{2}$ unknowns $U_{l}$,

$$
A_{l, 0} U_{l}=F_{l}
$$

where $(\otimes$ denotes the tensor product of spaces, operators and vectors)

$$
A_{l, 0}=\varepsilon c_{l}^{0} \otimes I+I \otimes c_{l}^{0}+I \otimes I
$$


is the Galerkin approximation (stiffness matrix) of $\mathscr{A}$ in $V_{l} \otimes V_{l}$. In (2.9) $c_{l}^{0}$ is a symmetric and circulant $n_{l} \times n_{1}$ matrix [8], which is completely determined by the connection coefficients $\Gamma_{k}^{0}(2.4)$,

$$
c_{l}^{0}=\delta_{l}^{-2}\left(\begin{array}{cccccccccccc}
\Gamma_{0}^{0} & \Gamma_{1}^{0} & \cdots & \Gamma_{p}^{0} & 0 & 0 & \cdots & 0 & \Gamma_{p}^{0} & \cdots & \Gamma_{2}^{0} & \Gamma_{1}^{0} \\
\Gamma_{1}^{0} & \Gamma_{0}^{0} & \cdots & \cdots & \Gamma_{p}^{0} & 0 & \cdots & 0 & 0 & \Gamma_{p}^{0} & \cdots & \Gamma_{2}^{0} \\
\vdots & \vdots & \vdots & \vdots & \vdots & \vdots & \vdots & \vdots & \vdots & \vdots & \vdots & \vdots \\
\Gamma_{1}^{0} & \cdots & \Gamma_{p}^{0} & 0 & 0 & 0 & \cdots & \Gamma_{p}^{0} & \cdots & \cdots & \Gamma_{1}^{0} & \Gamma_{0}^{0}
\end{array}\right),
$$

where $p=2 N-2$ and $\delta_{l}=2^{-l}$ is the discretization step-size. We denote a circulant matrix by its first row,

$$
c_{h}^{0}=\delta_{l}^{-2} \operatorname{Cir}_{n_{l}}\left(\begin{array}{llllllllll}
\Gamma_{0}^{0} & \Gamma_{1}^{0} & \cdots & \Gamma_{p}^{0} & 0 & \cdots & 0 & \Gamma_{p}^{0} & \cdots & \left.\Gamma_{1}^{0}\right) .
\end{array}\right.
$$

\section{Multilevel Scheme}

\subsection{Definitions and Notation}

For the definition of the FDMGM we supply some notation. A more detailed discussion of the wavelet variation of the FDMGM can be found in [16], see also the original paper [11]. First, we define the matrices $A_{l, m} \in \mathbf{R}^{n_{1}^{2} \times n_{l}^{2}}$ by

$$
A_{l, m}:=\varepsilon c_{l}^{m} \otimes I+I \otimes c_{l}^{0}+I \otimes I,
$$

where

$$
c_{l}^{m}=\delta_{l}^{-2} \operatorname{Cir}_{n_{l}}\left(\begin{array}{lllllllll}
\Gamma_{0}^{m} & \cdots & \Gamma_{2 N-2}^{m} & 0 & \cdots & 0 & \Gamma_{2 N-2}^{m} & \cdots & \Gamma_{1}^{m}
\end{array}\right)
$$

with the connection coefficients $\Gamma_{k}^{m}(2.4)$ of the wavelet packet $\psi^{m}(2.2)$ resp. (2.3). We have that

$$
A_{l-1,2 m}=(h \otimes h) A_{l, m}\left(h^{t} \otimes h^{t}\right) \quad \text { and } \quad A_{l-1,2 m+1}=(g \otimes h) A_{l, m}\left(g^{t} \otimes h^{t}\right),
$$

where $h$ and $g$ are the Mallat transformations (2.5) and (2.6) of appropriate dimension. All of the matrices $A_{l, m}$ are positive definite.

The iteration matrix of the basic iterative method (BIM) (also called smoothing iteration [10]) with respect to $A_{l, m}$ will be denoted by

$$
S_{l, m}=I-W_{l, m}^{-1} A_{l, m} \text {. }
$$

The matrix $W_{l, m}$ characterizes the special BIM, for instance the choice $W_{l, m}=$ $\vartheta_{l, m}^{-1} \operatorname{diag}\left(A_{l, m}\right), \vartheta_{l, m} \in \mathbf{R}$, gives the daped Jacobi iteration.

Now, we are able to formulate the recursive multilevel procedure. We let $L_{e} \geq 0$ be the coarsest level, that is the level where the corresponding linear systems are solved exactly, and we let $L$ be the level number with respect to the finest discretization. We have four variable quantities in our procedure: 
- $l$ is the varying level, $L_{e} \leq l \leq L$,

- $m$ is a non-negative integer on level $l, 0 \leq m \leq 2^{L-I}-1$,

- $w \in \mathbf{R}^{n_{l}^{2}}$ is a starting guess,

- $b \in \mathbf{R}^{n_{l}^{2}}$ is a right hand side or a defect.

We then define the following recursive multilevel procedure MLP:

$\operatorname{MLP}(l, m, w, b)$

begin

if $l=L_{e}$ then $w:=A_{l, m}^{-1} b$ else

$w:=\mathrm{S}_{l, m}^{v_{1}} w+\sum_{j=0}^{v_{1}-1} S_{l, m}^{j} W_{l, m}^{-1} b \quad\left(v_{1}\right.$ steps of the BIM $)$

$d:=A_{l, m} w-b, v_{0}=0, v_{1}=0$

$\operatorname{MLP}\left(l-1,2 m, v_{0},(h \otimes h) d\right)$

$\operatorname{MLP}\left(l-1,2 m+1, v_{1},(g \otimes h) d\right)$

$w:=w-\beta_{m}\left(h^{t} \otimes h^{t}\right) v_{0}-\beta_{m}\left(g^{t} \otimes h^{t}\right) v_{1}$

$w:=S_{l, m}^{v_{2}} w+\sum_{j=0}^{v_{2}-1} S_{l, m}^{j} W_{l, m}^{-1} b \quad\left(v_{2}\right.$ steps of the BIM)

end

The above procedure describes a damped $V$-cycle with damping parameters $\beta_{m} \geq \mathbf{R}$. One step of the multilevel method for solving (2.8) on level $L \geq 1$ is performed by

$$
\begin{gathered}
w:=U_{L}^{\mu}, \\
\operatorname{MLP}\left(L, 0, w, F_{L}\right), \\
U_{L}^{\mu+1}:=w .
\end{gathered}
$$

The $V$-cycle is an $O\left(N \cdot n_{L}^{2}\right)$-algorithm, see [11] and [16]. It is assumed that the connection coefficients are precomputed and stored. The precomputation of the connection coefficients $\left\{\Gamma_{k}^{m}\right\}_{m=0, \ldots, 2^{L}}$ requires the solution of a linear system of dimension $4 N-3$ for the $\Gamma_{\mu}^{0}$ 's [13]; the other connection coefficients are generated from these by Mallat transformations involving $O\left(2^{L} \cdot N^{2}\right)$ operations.

The convergence rates of the iteration (3.5) without damping $\left(\beta_{m} \equiv 1\right)$ for $L \in\{3,4,5\}$ with $L_{e}=0$ and for the Daubechies wavelet packets $N=3$ are plotted in Fig. 1 . We used the damped Jacobi iteration as BIM with damping parameters $\vartheta_{l, m}=$ $\Gamma_{0}^{m} / \sum_{k}\left|\Gamma_{k}^{m}\right|$, see [16] for an explanation, and with $v_{1}=2$ and $v_{2}=0$. The GaußSeidel relaxation as BIM gives the same qualitative behaviour of the convergence speed.

Next, we consider the iteration matrix $M_{L, 0}\left(v_{1}, v_{2}\right)$ of the iteration (3.5). Let $M_{l, m}\left(v_{1}, v_{2}\right)$ denote the iteration matrix of the iteration

$$
\begin{gathered}
w:=U_{l}^{\mu}, \\
\operatorname{MLP}\left(l, m, w, F_{l}\right), \\
U_{l}^{\mu+1}:=w,
\end{gathered}
$$

that is, we start our multigrid procedure to solve $A_{l, m} U_{l}=F_{l}$ on level $l$. 
Lemma 3.1. The above defined matrices $M_{l, m}\left(v_{1}, v_{2}\right), L_{e}<l \leq L$, for the iteration (3.6) satisfy the recursion

$$
\begin{aligned}
& M_{l, m}\left(v_{1}, v_{2}\right) \\
& =S_{l, m}^{v_{2}}\left(I-\beta_{m}\left(h^{t} \otimes h^{t}\right)\left\{I-M_{l-1,2 m}\left(v_{1}, v_{2}\right)\right\} A_{l-1,2 m}^{-1}(h \otimes h) A_{l, m}\right. \\
& \left.\quad-\beta_{m}\left(g^{t} \otimes h^{t}\right)\left\{I-M_{l-1,2 m+1}\left(v_{1}, v_{2}\right)\right\} A_{l-1,2 m+1}^{-1}(g \otimes h) A_{l, m}\right) S_{l, m}^{v_{1}}
\end{aligned}
$$

with $M_{L_{e}, m}\left(v_{1}, v_{2}\right)=0$.

Proof: The recursion (3.7) follows by transferring the proof of Lemma 7.1.4 in [10] to our situation.

\subsection{Robustness of the Damped V-Cycle}

For our considerations we will need the Euclidean (spectral) norm $\|w\|=\langle w, w\rangle^{1 / 2}$ as well as the energy-norms relative to the $A_{l, m}{ }^{\prime} \mathrm{s},\|w\|_{l, m}=\langle w, w\rangle_{l, m}^{1 / 2}:=\left\langle A_{l, m} w, w\right\rangle^{1 / 2}$. The associated matrix norms are denoted by the same symbols. With $\varrho(B)$ we abbreviate the spectral radius of $B$ which is the largest absolute value of the eigenvalues of $B$.

The following proposition has been proved in [16].

Proposition 3.2. There exist positive numbers $\sigma_{m}<1$ being independent of $\varepsilon$ and $\delta_{l}$ such that

$$
\varrho\left(A_{l-1,2 m}^{-1} B_{l, m} A_{l-1,2 m+1}^{-1} B_{l, m}^{t}\right) \leq \sigma_{m}
$$

with $B_{l, m}=(h \otimes h) A_{l, m}\left(g^{t} \otimes h^{t}\right)=s h c_{l}^{m} g^{t} \otimes I$. Further, the strong Cauchy inequality

$$
\left|\langle w, v\rangle_{l, m}\right| \leq \sqrt{\sigma_{m}}\|w\|_{l, m}\|v\|_{l, m}
$$

holds true for all $w, v \in \mathbf{R}^{n_{t}^{2}}$ satisfying $w=\left(h^{t} h \otimes h^{t} h\right) w$ and $v=\left(g^{t} g \otimes h^{t} h\right) v$, respectively.

The numbers $\sigma_{m}$ will play a critical role in our convergence analysis of the $V$-cycle presented in this section. They are uniformly bounded smaller than 1, i.e.

$$
\sigma:=\sup \left\{\sigma_{m} \mid m \in \mathbf{N}_{0}\right\}<1,
$$

which will be proved in Lemma A.2 (Appendix). Table 1 shows the first a few $\sigma_{m}$ 's for the wavelet orders $N=3$ and $N=4$.

Table 1. The numbers $\sigma_{m}$ for the Daubechies wavelets of order 3 and 4

\begin{tabular}{c|cccccccc}
\hline & $\sigma_{0}$ & $\sigma_{1}$ & $\sigma_{2}$ & $\sigma_{3}$ & $\sigma_{4}$ & $\sigma_{5}$ & $\sigma_{6}$ & $\sigma_{7}$ \\
\hline$N=3$ & $2.9 \mathrm{E}-1$ & $2.8 \mathrm{E}-2$ & $2.4 \mathrm{E}-3$ & $2.3 \mathrm{E}-2$ & $1.9 \mathrm{E}-4$ & $1.6 \mathrm{E}-3$ & $5.5 \mathrm{E}-3$ & $5.0 \mathrm{E}-3$ \\
$N=4$ & $1.9 \mathrm{E}-1$ & $8.5 \mathrm{E}-3$ & $6.9 \mathrm{E}-4$ & $6.3 \mathrm{E}-3$ & $5.0 \mathrm{E}-5$ & $5.1 \mathrm{E}-4$ & $1.3 \mathrm{E}-3$ & $1.4 \mathrm{E}-4$ \\
\hline
\end{tabular}


From now on we require a symmetry condition for our BIM, that is, the matrix $W_{l, m}$ in (3.3) satisfies

$$
W_{l, m}^{t}=W_{l, m} \geq A_{l, m} \quad \text { and } \quad\left\|W_{l, m}\right\| \leq C_{W} \delta_{l}^{-2} .
$$

The notation $A \leq B$ signifies that $A$ and $B$ are symmetric matrices and that $B-A$ is positive semi-definite.

Remark. The (sufficiently) damped Jacobi iteration as well as the symmetric GaußSeidel iteration fulfill (3.10), see e.g. [10].

We introduce the abbreviations $p_{0}=h \otimes h, p_{1}=g \otimes h$ and the transformed matrices

$$
\begin{gathered}
\hat{M}_{l, m}\left(v_{1}, v_{2}\right)=A_{l, m}^{1 / 2} M_{l, m}\left(v_{1}, v_{2}\right) A_{l, m}^{-1 / 2}, \quad \hat{p}_{i}=A_{l-1,2 m+i}^{-1 / 2} p_{i} A_{l, m}^{1 / 2}, \quad i=0,1, \\
\hat{S}_{l, m}=A_{l, m}^{1 / 2} S_{l, m} A_{l, m}^{-1 / 2}=I-X_{l, m} \quad \text { with } \quad X_{l, m}=A_{l, m}^{1 / 2} W_{l, m}^{-1} A_{l, m}^{1 / 2} .
\end{gathered}
$$

The recursion (3.7) implies

$$
\hat{M}_{l, m}\left(v_{1}, v_{2}\right)=\hat{S}_{l, m}^{v_{2}}\left(I-\beta_{m} \sum_{i=0}^{1} \hat{p}_{i}^{\tau}\left\{I-\hat{M}_{l-1,2 m+i}\left(v_{1}, v_{2}\right)\right\} \hat{p}_{i}\right) \hat{S}_{l, m}^{v_{1}} .
$$

Before we formulate the robustness result for the damped $V$-cycle in Theorem 3.8 we first supply some preparatory lemmas and corollaries.

Lemma 3.3. If $\beta_{m} \in\left[0,\left(1+\sqrt{\sigma_{m}}\right)^{-1}\right]$ then

$$
0 \leq Q_{l, m}:=I-\beta_{m}\left(\hat{p}_{0}^{t} \hat{p}_{0}+\hat{p}_{1}^{t} \hat{p}_{1}\right) \leq I .
$$

Proof: The relation (3.11) is equivalent to $0 \leq \beta_{m}\left(\hat{p}_{0}^{t} \hat{p}_{0}+\hat{p}_{1}^{t} \hat{p}_{1}\right) \leq I$ which is satisfied if $\beta_{m} \in\left[0, \varrho\left(\hat{p}_{0}^{t} \hat{p}_{0}+\hat{p}_{1}^{t} \hat{p}_{1}\right)^{-1}\right]$ because $\hat{p}_{0}^{t} \hat{p}_{0}+\hat{p}_{1}^{t} \hat{p}_{1}$ is positive semidefinite. We have that $\hat{p}_{0}^{t} \hat{p}_{0}+\hat{p}_{1}^{t} \hat{p}_{1}=A_{l, m}^{1 / 2} U^{t} D^{-1} U A_{l, m}^{1 / 2}$ with the $2 \times 2$-block diagonal matrix $D=\operatorname{diag}\left(A_{l-1,2 m}, A_{l-1,2 m+1}\right)$ and with the mapping $U: \mathbf{R}^{n_{l}^{2}} \rightarrow \mathbf{R}^{n_{i}^{2} / 2}$ defined by

$$
U:=\left(\begin{array}{c}
p_{0} \\
p_{1}
\end{array}\right)=\left(\begin{array}{c}
h \otimes h \\
g \otimes h
\end{array}\right) .
$$

Now, let us estimate the spectral radius $\varrho\left(A_{l, m}^{1 / 2} U^{t} D^{-1} U A_{l, m}^{1 / 2}\right)$ :

$$
\begin{aligned}
\varrho\left(A_{l, m}^{1 / 2} U^{t} D^{-1} U A_{l, m}^{1,2}\right) & =\varrho\left(D^{-1} U A_{l, m} U^{t}\right)=\varrho\left(I-\left(I-D^{-1} U A_{l, m} U^{t}\right)\right) \\
& \leq 1+\varrho\left(I-D^{-1} U A_{l, m} U^{t}\right) .
\end{aligned}
$$

The estimate (3.8) in Proposition 3.2 implies that

$$
r_{m}\left(\varepsilon, \delta_{l}\right):=\varrho\left(I-D^{-1} U A_{l, m} U^{t}\right) \leq \sqrt{\sigma_{m}} .
$$

Lemma 3.4. Suppose (3.10) and let $\widetilde{Q}_{l, m}:=I-A_{l, m}^{1 / 2} U^{t}\left(U A_{l, m} U^{t}\right)^{-1} U A_{l, m}^{1 / 2}$. Then,

$$
0 \leq \widetilde{Q}_{l, m} \leq C X_{i, m} \text { s }
$$

where $C$ is a constant being independent of $\varepsilon, \delta_{l}$ and $m$.

Proof: We follow the proof of Lemma 6.4.6 in [10]. The statement of Lemma B.1 (Appendix) implies $\widetilde{Q}_{l, m} \leq C_{A} \delta_{l}^{2} A_{l, m}$ and (3.10) can be rewritten as $I \leq C_{W} \delta_{l}^{-2} W_{l, m}^{-1}$ which gives $A_{l, m} \leq C_{W} \delta_{l}^{-2} X_{l, m}$. Hence, (3.14) holds with $C=C_{A} C_{W}$. 
Corollary 3.5. Suppose (3.10) and let $\beta_{m} \in\left[0,\left(1+\sqrt{\sigma_{m}}\right)^{-1}\right]$. Then,

$$
0 \leq Q_{l, m} \leq C X_{l, m}+d_{m} I
$$

where $d_{m}=1-\beta_{m}\left(1-\sqrt{\sigma_{m}}\right)$ and where $C$ is the constant from (3.14).

Proof: We have that $Q_{l, m}-\widetilde{Q}_{l, m}=A_{l, m}^{1 / 2} U^{t}\left(\left(U A_{l, m} U^{t}\right)^{-1}-\beta_{m} D^{-1}\right) U A_{l, m}^{1 / 2}$ with $U$ and $D$ as in the proof of Lemma 3.3. Further,

$$
\begin{aligned}
\varrho\left(Q_{l, m}-\tilde{Q}_{l, m}\right) & =\varrho\left(I-\beta_{m} D^{-1} U A_{l, m} U^{t}\right) \\
& =\varrho\left(\left(1-\beta_{m}\right) I+\beta_{m}\left(I-D^{-1} U A_{l, m} U^{t}\right)\right) \\
& \leq 1-\beta_{m}+\beta_{m} \varrho\left(I-D^{-1} U A_{l, m} U^{t}\right) \\
& \leq 1-\beta_{m}+\beta_{m} \sqrt{\sigma_{m}}=d_{m},
\end{aligned}
$$

where we used (3.13). From (3.14) follows that

$$
\begin{aligned}
0 \leq Q_{l, m} & \leq \widetilde{Q}_{l, m}+\varrho\left(Q_{l, m}-\widetilde{Q}_{l, m}\right) I \\
& \leq C X_{l, m}+d_{m} I .
\end{aligned}
$$

We set $\hat{M}_{l, m}:=\hat{M}_{l, m}(v / 2, v / 2)$ which formally makes sense for any real $v \geq 0$.

Lemma 3.6. Suppose (3.10). Let $\beta_{m} \in\left[0,\left(1+\sqrt{\sigma_{m}}\right)^{-1}\right]$ and let $d_{m}=1-\beta_{m}\left(1-\sqrt{\sigma_{m}}\right)$. If

$$
0 \leq \hat{M}_{l-1,2 m+i} \leq \xi_{l-1,2 m+i} I, \quad 0 \leq \xi_{l-1,2 m+i} \leq 1, \quad i=0,1,
$$

then

$$
0 \leq \hat{M}_{l, m} \leq \xi_{l, m} I, \quad \xi_{l, m}=\min _{d_{m}\left(1-\bar{\xi}_{l, m}\right)+\bar{\xi}_{l, m} \leq y \leq 1} \max _{0 \leq x \leq 1} f(x, y),
$$

with $\bar{\xi}_{l, m}=\max \left\{\xi_{l-1,2 m}, \xi_{l-1,2 m+1}\right\}$ and $f(x, y)=(1-x)^{v}\left(y+(1-y) C x /\left(1-d_{m}\right)\right)$ where $C$ is the constant from (3.14).

Proof: The proof is similar to the proof of Lemma 7.2.1 in [10].

Since $\hat{M}_{l, m}=\hat{S}_{l, m}^{v / 2} Q_{l, m} \hat{S}_{l, m}^{v / 2}+\beta_{m} \hat{S}_{l, m}^{v / 2} \sum_{i=0}^{1} \hat{p}_{i}^{t} \hat{M}_{l-1,2 m+i} \hat{p}_{i} \hat{S}_{l, m}^{v / 2}$ and since $\hat{M}_{l-1,2 m+i} \geq 0$, $i=0,1,(3.11)$ proves $M_{l, m} \geq 0$. Using (3.16) and (3.11) we obtain

$$
\begin{aligned}
\hat{M}_{l, m} & \leq \hat{S}_{l, m}^{v / 2}\left\{I-\beta_{m} \hat{p}_{0}^{t} \hat{p}_{0}\left(1-\xi_{l-1,2 m}\right)-\beta_{m} \hat{p}_{1}^{t} \hat{p}_{1}\left(1-\xi_{l-1,2 m+1}\right)\right\} \hat{S}_{l, m}^{v / 2} \\
& \leq \hat{S}_{l, m}^{v / 2}\left\{I-\left(1-\bar{\xi}_{l, m}\right) \beta_{m}\left(\hat{p}_{0}^{t} \hat{p}_{0}+\hat{p}_{1}^{t} \hat{p}_{1}\right)\right\} \hat{S}_{l, m}^{v / 2} \\
& =\hat{S}_{l, m}^{v / 2}\left\{\bar{\xi}_{l, m} I+\left(1-\bar{\xi}_{l, m}\right) Q_{m, l}\right\} \hat{S}_{l, m}^{v / 2}:
\end{aligned}
$$

By (3.15) and (3.11) we get $0 \leq Q_{l, m} \leq \alpha C X_{l, m}+\left(\alpha d_{m}+(1-\alpha)\right) I$ for all $\alpha \in[0,1]$. Hence,

$$
\begin{aligned}
\widehat{M}_{l, m} & \leq \widehat{S}_{l, m}^{v / 2}\left\{\alpha\left(1-\bar{\xi}_{l, m}\right) C X_{l, m}+\left(1-\alpha\left(1-\bar{\xi}_{l, m}\right)\left(1-d_{m}\right)\right) I\right\} \hat{S}_{l, m}^{v / 2} \\
& =\widehat{S}_{l, m}^{v / 2}\left(\frac{1-y}{1-d_{m}} C X_{l, m}+y I\right) \hat{S}_{l, m}^{v / 2}
\end{aligned}
$$

for all $y \in\left[d_{m}\left(1-\bar{\xi}_{l, m}\right)+\bar{\xi}_{l, m}, 1\right]$. The statement (3.17) follows by $0 \leq X_{l, m} \leq I$. 
Corollary 3.7. We adopt the assumptions and notations of Lemma 3.6. Further, let $\beta_{m}=\left(1+\sqrt{\sigma_{m}}\right)^{-1}$. Then, there exists a positive integer $\bar{v}$ independent of $\varepsilon, \delta_{l}$ and $m$ such that

$$
\begin{aligned}
\varrho\left(\hat{M}_{l, m}\right) \leq \xi_{l, m} & =1-\frac{1-\sqrt{\sigma_{m}}}{1+\sqrt{\sigma_{m}}} \min \left\{1-\xi_{l-1,2 m}, 1-\xi_{l-1,2 m+1}\right\} \\
& =1-\frac{1-\sqrt{\sigma_{m}}}{1+\sqrt{\sigma_{m}}}\left(1-\max \left\{\xi_{l-1,2 m}, \xi_{l-1,2 m+1}\right\}\right)
\end{aligned}
$$

for all $v \geq \vec{v}$.

Proof: The partial derivative $f_{x}(x, y)=(1-x)^{v-1}\left(-(v+1)(1-y) C x /\left(1-d_{m}\right)-\right.$ $\left.v y+(1-y) C /\left(1-d_{m}\right)\right)$ shows that $f_{x}(x, y) \leq 0$ for $y \in\left[d_{m}\left(1-\bar{\xi}_{l, m}\right)+\bar{\xi}_{l, m}, 1\right]$ if $v \geq \bar{v}$ with $\bar{v}$ sufficiently large. The lower bound $\bar{v}$ does not depend on $m$ because the $\sigma_{m}$ 's are uniformly bounded smaller than 1, see (3.9). The function $f$ is monotonically decreasing in $x$. Using the statement of Lemma 3.6 we estimate

$$
\begin{aligned}
\xi_{l, m} & =\min _{d_{m}\left(1-\bar{\xi}_{l, m}\right)+\bar{\xi}_{l, m} \leq y \leq 1} f(0, y)=\min _{d_{m}\left(1-\bar{\xi}_{l, m}\right)+\bar{\xi}_{l, m} \leq y \leq 1} y \\
& =1-\beta_{m}\left(1-\sqrt{\sigma_{m}}\right)\left(1-\bar{\xi}_{l, m}\right) .
\end{aligned}
$$

Now, we are able to formulate and to prove our main result which shows that the convergence rate of the damped $V$-cycle of the FDMGM depends at most on the number of levels.

Theorem 3.8. Let $M_{L, 0}(v, v)$ be the iteration matrix of the iteration process (3.5) with damping factors $\beta_{m}=\left(1+\sqrt{\sigma_{m}}\right)^{-1}$. Further, let $\bar{v}$ be the positive integer determined in Corollary 3.7. If $2 v \geq \bar{v}$ then

$$
\varrho\left(M_{L, 0}(v, v)\right)=\left\|M_{L, 0}(v, v)\right\|_{L, 0} \leq 1-\left(\frac{1-\sqrt{\sigma}}{1+\sqrt{\sigma}}\right)^{L-L_{e}},
$$

where $0 \leq \sigma<1$ is defined in (3.9).

Proof: We have that $\varrho\left(M_{l, m}(v, v)\right)=\varrho\left(\hat{M}_{l, m}(v, v)\right)=\left\|M_{l, m}(v, v)\right\|_{l, m}$. Since $\left(1-\sqrt{\sigma_{m}}\right) /$ $\left(1+\sqrt{\sigma_{m}}\right) \geq(1-\sqrt{\sigma}) /(1+\sqrt{\sigma})$ for all $m$ and since $\xi_{L_{e}, m}=0,0 \leq m \leq 2^{L-L_{e}}-1$, an inductive application of Corollary 3.7 proves Theorem 3.8 .

Remark: The statement of Theorem 3.8 holds also true if we damp uniformly, i.e. $\beta_{m} \equiv(1+\sqrt{\sigma})^{-1}$.

We have numerical evidence that $\sigma=\sigma_{0}$ holds true, e.g. see Table 1.

In Fig. 2 the convergence rates of the FDMGM (3.5) are plotted for $L \in\{3,4,5\}$ with $L_{e}=0$ and for the uniform damping parameter $\beta=\beta_{m}=0.9$. The underlying Daubechies wavelet packets have order $N=3$. Again, the damped Jacobi iteration was used as BIM with the damping parameters described in Section 3.1 and with $v_{1}=2, v_{2}=0$. The choice $v_{1}=v_{2}=1$ leads to the same convergencer rates. If we replace the Jacobi iteration by the symmetric Gauß-Seidel iteration then we have better convegence rates. However, the dependence on the levels remains unchanged. 


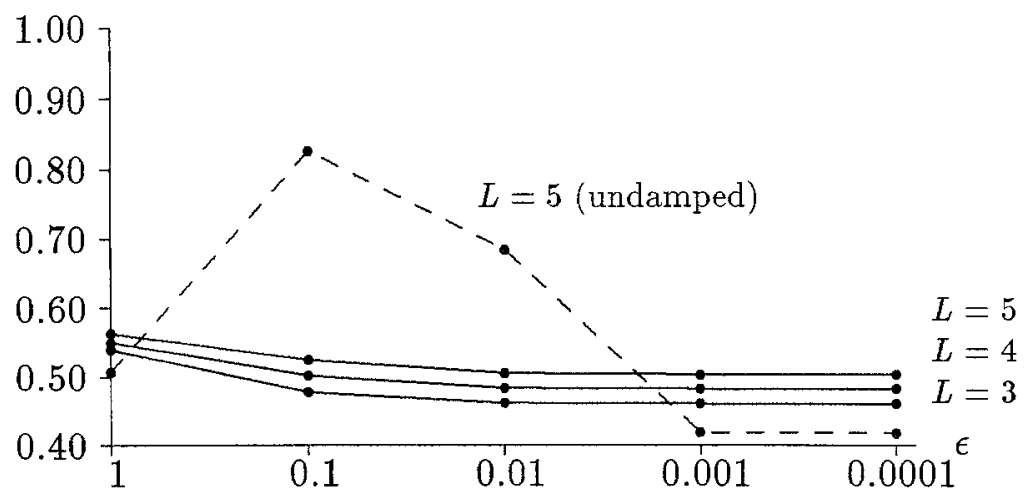

Figure 2. Damped V-cycle convergence rates of the wavelet variation of the FDMGM for different discretization step-sizes and for a uniform damping parameter $\beta=0.9$

In principle, one can compute reasonable approximations to the $\beta_{m}$ 's by explicit formulas for the $\sigma_{m}$ 's, see [16], in an efficient way. This parameter choice gives worse convergence rates than the presented rates for the uniform damping parameter $\beta=0.9$ which is larger than the maximum $\beta_{0}=0.65$ of the $\beta_{m}$ 's for $N=3$. One reason for this behaviour we find in (3.13) where we see that an optimal damping parameter should depend on $m, \varepsilon$ and $\delta_{l}$. For instance, if $\delta_{l}$ is fixed then $r_{m}\left(\varepsilon, \delta_{l}\right)$ tends to zero as $\varepsilon \rightarrow 0$. Further, an explicit expression of $r_{m}$ given in [16] shows that the assumption $r_{m}\left(\varepsilon, \delta_{l}\right)=0$ is reasonable if $\varepsilon \ll \delta_{l}^{2}$. Moreover, in the extreme case $\varepsilon=0$ where no damping is needed the proofs in Chapter 7.2 of [10] can be transfered to the FDMGM without any modifications and yield the contraction number

$$
\left\|M_{L, 0}\left(v_{1}, v_{2}\right)\right\|_{L, 0} \leq \frac{C}{\sqrt{C+v_{1}} \sqrt{C+v_{2}}} .
$$

\section{Conclusion}

In this paper, we showed that the convergence rate of the sufficiently damped $V$-cycle of the wavelet variation of the FDMGM depends at most on the number of levels (Theorem 3.8). This is a first $V$-cycle convergence result for the FDMGM introduced in [11] and [12]. The presented result might be improved by chosing another damping strategy than (3.4), that is, both branches of the coarse grid correction are damped separately, and by investigating the dependence of the damping parameters on $m, \varepsilon$ and $\delta_{l}$ more carefully.

\section{Appendix}

\section{A. Uniform Boundedness of the $\sigma_{m}$ 's}

In this Appendix the proof of (3.9) will be given. 
We define

$$
H(\omega)=\frac{1}{2} \cdot \sum_{k=0}^{2 N-1} a_{k} e^{-i k \omega}, \quad i=\sqrt{-1},
$$

with the coefficients $a_{k}$ from (2.1). The trigonometric polynomial $H$ satisfies

$$
H^{2}(\omega)+H^{2}(\omega+\pi)=1,
$$

see [6] or [7]. Further we will need the positive cosine series

$$
\lambda^{m}(\omega)=\Gamma_{0}^{m}+2 \sum_{k=1}^{2 N-2} \Gamma_{k}^{m} \cos (k \omega)
$$

with the second order connection coefficients $\Gamma_{k}^{m}(2.4)$. We have the following recursion formula

$$
\begin{aligned}
\lambda^{2 m}(\omega) & =4\left(|H(\omega / 2)|^{2} \lambda^{m}(\omega / 2)+|H(\omega / 2+\pi)|^{2} \lambda^{m}(\omega / 2+\pi)\right), \\
\lambda^{2 m+1}(\omega) & =4\left(|H(\omega / 2+\pi)|^{2} \lambda^{m}(\omega / 2)+|H(\omega / 2)|^{2} \lambda^{m}(\omega / 2+\pi)\right),
\end{aligned}
$$

see [16]. With these definitions we are able to express the numbers $\sigma_{m}$ from Proposition 3.2 by

$$
\sigma_{m}=\sup _{0 \leq \omega \leq 2 \pi} \frac{R^{m}(\omega)}{R^{m}(\omega)+\lambda^{m}(\omega / 2) \lambda^{m}(\omega / 2+\pi)}
$$

where $R^{m}(\omega)=|H(\omega / 2)|^{2}|H(\omega / 2+\pi)|^{2}\left(\lambda^{m}(\omega / 2)-\lambda^{m}(\omega / 2+\pi)\right)^{2}$, see also [16].

In the lemma below the bracket expression ['] denotes the 'greatest integer' and ld denotes the logarithm with respect to the basis 2 .

Lemma A.1. The inclusion

$$
0<\min _{0 \leq \omega \leq 2 \pi} \lambda^{1}(\omega) \leq 4^{-[1 \mathrm{~d} m]} \lambda^{m}(\omega) \leq \max _{0 \leq \omega \leq 2 \pi} \lambda^{1}(\omega)<\infty
$$

holds true for all $m \geq 1$.

Proof: Both inner inequalities follow inductively from (A.4), (A.5) and (A.2). The leftmost inequality was shown in Lemma 2.3 (iv) in [16].

Lemma A.2. The supremum of the $\sigma_{m}$ 's (A.6) is bounded smaller than 1,

$$
\sup \left\{\sigma_{m} \mid m \in \mathbf{N}_{0}\right\}<1 \text {. }
$$

Proof: We assume that $\sup \left\{\sigma_{m} \mid m \geq 1\right\}=1$. Then, for any $\alpha>0$ there exists an integer $m_{\alpha} \geq 1$ and an $\omega_{\alpha} \in[0,2 \pi)$ such that

$$
\sigma_{m_{\alpha}}=\frac{R^{m_{\alpha}}\left(\omega_{\alpha}\right)}{R^{m_{\alpha}}\left(\omega_{\alpha}\right)+\lambda^{m_{\alpha}}\left(\omega_{\alpha} / 2\right) \lambda^{m_{\alpha}}\left(\omega_{\alpha} / 2+\pi\right)} \geq 1-\alpha
$$

which is equivalent to

$$
\alpha \geq \frac{16^{-\left[\mathrm{ld} m_{\alpha}\right] \lambda^{m_{\alpha}}}\left(\omega_{\alpha} / 2\right) \lambda^{m_{\alpha}}\left(\omega_{\alpha} / 2+\pi\right)}{16^{-\left[1 \mathrm{~d} m_{\alpha}\right]} R^{m_{\alpha}}\left(\omega_{\alpha}\right)+16^{-\left[\mathrm{ld} m_{\alpha}\right]} \lambda^{m_{\alpha}}\left(\omega_{\alpha} / 2\right) \lambda^{m_{\alpha}}\left(\omega_{\alpha} / 2+\pi\right)}>0 .
$$


By (A.7) and by $|H(\omega / 2)|^{2}|H(\omega / 2+\pi)|^{2}<1 / 4$ which follows from (A.2) we derive $0<16^{-\left[\mathrm{Id} m_{\alpha}\right]} R^{m_{\alpha}}\left(\omega_{\alpha}\right) \leq\left(\max _{0 \leq \omega \leq 2 \pi} \lambda^{1}(\omega)\right)^{2}$. Therefore, (A.8) implies that

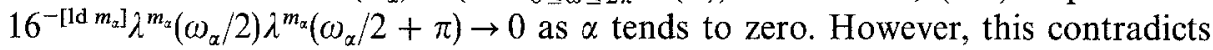
$4^{-[\mathrm{ld} m]} \lambda^{m}(\omega) \geq \min _{0 \leq \omega \leq 2 \pi} \lambda^{1}(\omega)>0$, see (A.7). Hence, $\sup \left\{\sigma_{m} \mid m \geq 1\right\}<1$. Since $\sigma_{0}<1$ Lemma A. 2 is proved.

\section{B. An Approximation Property}

Our goal is to verify the approximation property stated in Lemma B.1.

Lemma B.1. Let the matrices $A_{l, m}$ be defined as in (3.1) and the mapping $U$ as in (3.12). Then,

$$
\left\|A_{l, m}^{-1}-U^{t}\left(U A_{l, m} U^{t}\right)^{-1} U\right\| \leq C_{A} \delta_{l}^{-2},
$$

where $C_{A}$ is a constant being independent of $\varepsilon_{,} \varepsilon_{l}$ and $m$.

First we note that $\lambda_{\mu}^{m}=\delta_{l}^{-2} \lambda^{m}\left(2 \pi / \mu / n_{l}\right), \mu=0, \ldots, n_{l}-1$, are the eigenvalues of $c_{l}^{m}$ (3.2) where the function $\lambda^{m}$ is given in (A.3). All circulant matrices of the dimension $n_{l}$ share the same system of $l^{2}$-orthonormal eigenvectors $v_{\mu}, \mu=0, \ldots, n_{l}-1$,

$$
\left(v_{\mu}\right)_{k}=\frac{1}{\sqrt{n_{l}}} e^{-i 2 \pi \mu k / n_{l}},
$$

see [8]. Consequently, the matrices $A_{l, m}$ have the eigenvalues

$$
\Lambda_{m ; \mu, v}=\delta_{l}^{-2}\left(\varepsilon \lambda^{m}\left(2 \pi \mu / n_{l}\right)+\lambda^{0}\left(2 \pi v / n_{l}\right)\right)+1,
$$

$0 \leq \mu, v \leq n_{l}-1$, with corresponding eigenvectors $v_{\mu, v}=v_{\mu} \otimes v_{v}$.

Lemma B.2. We have that

$$
\left\|U^{t}\left(U A_{l, m} U^{t}\right)^{-1} U A_{l, m}\right\| \leq C,
$$

where the constant $C$ does not depend on $\varepsilon, \delta_{l}$ or $m$.

Proof: The proof will be very technical and we will use results proved in [16].

The matrix $U A_{l, m} U^{t}$ has the following block structure

$$
U A_{l, m} U^{t}=\left(\begin{array}{cc}
A_{l-1,2 m} & B \\
B^{t} & A_{l-1,2 m+1}
\end{array}\right)
$$

with $B=\varepsilon c_{l-1}^{m, c} \otimes I$ and $c_{l-1}^{m, c}=h c_{l}^{m} g^{t}$. Again, $c_{l-1}^{m, c}$ is a circulant matrix,

$$
c_{l-1}^{m, c}=\delta_{l-1}^{-2} \operatorname{Cir}_{n_{l-1}}\left(\begin{array}{lllllllllll}
\Gamma_{0}^{m, c} & \Gamma_{1}^{m, c} & \cdots & \Gamma_{2 N-2}^{m, c} & 0 & \cdots & 0 & \Gamma_{2-2 N}^{m, c} & \cdots & \Gamma_{-1}^{m, c}
\end{array}\right),
$$

determined by the (mixed) connection coefficients

$$
\Gamma_{k}^{m, c}=\int_{\mathbf{R}}\left(\psi^{2 m}\right)^{\prime}(x-k)\left(\psi^{2 m+1}\right)^{\prime}(x) d x .
$$

The eigenvalues of $c_{l-1}^{m, c}$ are denoted by $\lambda_{\mu}^{m, c}=\delta_{l-1}^{-2} \lambda^{m, c}\left(2 \pi \mu / n_{l-1}\right), 0 \leq \mu \leq n_{l-1}-1$, where $\lambda^{m, c}(\omega)=\sum_{k=2-2 N}^{2 N-2} \Gamma_{k}^{m, c} e^{-i k \omega}$ satisfying 


$$
\lambda^{m, c}(\omega)=H(\omega / 2) \overline{H(\omega / 2+\pi)}\left(\lambda^{m}(\omega / 2)-\lambda^{m}(\omega / 2+\pi)\right),
$$

with $H$ as in (A.1). The inverse of $U A_{l, m} U^{t}$ can be expressed by its block entries

$$
\left(U A_{l, m} U^{t}\right)^{-1}=\left(\begin{array}{cc}
A_{0}^{-1}\left(I-B A_{1}^{-1} \Sigma^{-1} B^{t} A_{0}^{-1}\right) & -A_{0}^{-1} B A_{1}^{-1} \Sigma^{-1} \\
-A_{1}^{-1} \Sigma^{-1} B^{t} A_{0}^{-1} & A_{1}^{-1} \Sigma^{-1}
\end{array}\right)
$$

where $\Sigma=I-A_{1}^{-1} B^{t} A_{0}^{-1} B$. For convenience we set $A_{0}:=A_{l-1,2 m}$ and $A_{1}:=$ $A_{l-1,2 m+1}$. The matrix $\Sigma$ is invertible because

$$
\varrho(I-\Sigma) \leq \sigma<1
$$

due to (3.8) and (3.9). Let $f$ be in $\mathbf{R}^{n^{2}}$. Then,

$$
\begin{aligned}
\left\|U^{t}\left(U A_{l, m} U^{t}\right)^{-1} U A_{l, m} f\right\|^{2}= & \sum_{\mu, v} \sum_{\alpha, \beta} A_{m ; \mu, v} A_{m ; \alpha, \beta} \\
& \times\left\langle\left(U A_{l, m} U^{t}\right)^{-1} U v_{\mu, v},\left(U A_{l, m} U^{t}\right)^{-1} U \bar{v}_{\alpha, \beta}\right\rangle\left\langle f, v_{\mu, v}\right\rangle \\
& \times\left\langle f, \bar{v}_{\alpha, \beta}\right\rangle .
\end{aligned}
$$

A simple calculation shows that

$$
U v_{\mu, v}=\left(\begin{array}{l}
H\left(\omega_{\mu}\right) H\left(\omega_{v}\right) \tilde{v}_{2 \mu, 2 v} \\
G\left(\omega_{\mu}\right) H\left(\omega_{v}\right) \tilde{v}_{2 \mu, 2 v}
\end{array}\right)
$$

Here, $\omega_{\mu}=2 \pi \mu / n_{l}$ and $G(\omega)=2^{-1} \sum_{k=0}^{2 N-1} b_{k} e^{-i k \omega}$ with the $b_{k}$ 's from (2.3). The vectors $\tilde{v}_{2 \mu, 2 v}$ are tensor products, $\tilde{v}_{2 \mu, 2 v}=\tilde{v}_{2 \mu} \otimes \tilde{v}_{2 v}$, where $\left(\tilde{v}_{2 \mu}\right)_{k}=\sqrt{2} e^{-i 2 \pi 2 \mu k i n_{1}} / \sqrt{n_{l}}$, $k=0, \ldots, n_{l-1}-1$. Note that $\tilde{v}_{2 \mu, 2 v}$ is an eigenvector of the circulant matrices $A_{0}$, $A_{1}, B$ and $B^{t}$, respectively. Using (B.4) as well as (B.2) leads to

$$
\left(U A_{l, m} U^{t}\right)^{-1} U v_{\mu, v}=\left(\begin{array}{c}
r_{\mu, v} \tilde{v}_{2 \mu, 2 v} \\
t_{\mu, v} \tilde{v}_{2 \mu, 2 v}
\end{array}\right)
$$

with

$$
\begin{aligned}
r_{\mu, v} & =H\left(\omega_{\mu}\right) H\left(\omega_{v}\right) \Lambda_{2 m ; 2 \mu, 2 v}^{-1}\left(1+\Lambda_{\Sigma ; 2 \mu, 2 v}^{-1}\right. \\
& \left.\times\left(\Lambda_{2 m+1 ; 2 \mu, 2 v}^{-1}\left|\Lambda_{2 m ; 2 \mu, 2 v}^{c}\right|^{2} \Lambda_{2 m ; 2 \mu, 2 v}^{-1}-\Lambda_{2 m ; 2 \mu, 2 v}^{c} \Lambda_{2 m+1 ; 2 \mu, 2 v}^{-1}\right)\right), \\
t_{\mu, v} & =G\left(\omega_{\mu}\right) H\left(\omega_{v}\right) \Lambda_{2 m+1 ; 2 \mu, 2 v}^{-1} \Lambda_{\Sigma ; 2 \mu, 2 v}^{-1}\left(-\bar{A}_{2 m ; 2 \mu, 2 v}^{c} \Lambda_{2 m ; 2 \mu, 2 v}^{-1}+1\right),
\end{aligned}
$$

where $A_{2 m ; 2 \mu, 2 v}^{c}=\varepsilon \delta_{l-1}^{-2} \lambda^{m, c}\left(2 \omega_{\mu}\right)$ and $A_{\Sigma ; 2 \mu, 2 v}=1-A_{2 m ; 2 \mu, 2 v}^{-1}\left|\Lambda_{2 m ; 2 \mu, 2 v}^{c}\right|^{2} \Delta_{2 m+1 ; 2 \mu, 2 v}^{-1}$. Since

$$
\begin{aligned}
\left\langle\left(U A_{l, m} U^{t}\right)^{-1} U v_{\mu, v},\left(U A_{l, m} U^{t}\right)^{-1} U \bar{v}_{\alpha, \beta}\right\rangle & =\left(r_{\mu, v} \bar{r}_{\alpha, \beta}+t_{\mu, v} \bar{t}_{\alpha, \beta}\right)\left\langle\tilde{v}_{2 \mu, 2 v}, \overline{\tilde{v}}_{2 \alpha, 2 \beta}\right\rangle \\
& =\left(r_{\mu, v} \bar{r}_{\alpha, \beta}+t_{\mu, v} \bar{t}_{\alpha, \beta}\right) A_{n_{t}}^{\mu, \alpha} \Delta_{n_{1}}^{v, \beta}
\end{aligned}
$$

(here $\Delta_{n_{l}}^{\mu, \alpha}=1$ if $|\mu-\alpha|=k n_{l} / 2, k \in \mathbf{Z}$, ad $\Delta_{n_{l}}^{\mu, \alpha}=0$ otherwise) we have that

$$
\left\|U^{t}\left(U A_{l, m} U^{t}\right)^{-1} U A_{l, m} f\right\| \leq 3\|f\| \max _{\mu, v} A_{m ; \mu, \nu}\left(\left|r_{\mu, v}\right|+\left|t_{\mu, \nu}\right|\right) .
$$

In the last step of the proof we show that the maximum is independent of $\varepsilon, \delta_{l}$ and $m$. Therefore we supply a bunch of estimates. First,

$$
\Lambda_{\Sigma ; 2 \mu, 2 v} \geq 1-\sigma \quad \text { and } \Lambda_{2 m ; 2 \mu, 2 v}^{-1}\left|\Lambda_{2 m ; 2 \mu, 2 v}^{c}\right|^{2} \Lambda_{2 m+1 ; 2 \mu, 2 v}^{-1} \leq \sigma
$$


by (B.3) and (3.8), (3.9), respectively. Further,

$$
\begin{aligned}
\frac{\left|H\left(\omega_{\mu}\right) H\left(\omega_{v}\right)\right| A_{m ; \mu, v}}{A_{2 m ; 2 \mu, 2 v}} & \leq 4 \sup _{0 \leq \omega, \eta \leq 2 \pi} \frac{|H(\omega) H(\eta)|\left(\varepsilon \lambda^{m}(\omega)+\lambda^{0}(\eta)+\delta_{l}^{2}\right)}{\varepsilon \lambda^{2 m}(2 \omega)+\lambda^{0}(2 \eta)+\delta_{l-1}^{2}} \\
& \leq 4 \max \{\underbrace{\left\{\sup _{0 \leq \omega \leq 2 \pi} \frac{|H(\omega)| \lambda^{m}(\omega)}{\lambda^{2 m}(2 \omega)},\right.}_{=E_{1}} \underbrace{\sup _{0 \leq \eta \leq 2 \pi} \frac{|H(\eta)| \lambda^{0}(\eta)}{\lambda^{0}(2 \eta)}}_{=E_{2}}, \frac{\delta_{l}^{2}}{\delta_{l-1}^{2}}\} .
\end{aligned}
$$

If $m \geq 1$ then (A.7) implies that $E_{1}$ is uniformly bounded in $m$. For $m=0, E_{1}$ equals $E_{2}$. Due to part (ii) of Lemma 2.3 in [16] $\lambda^{0}\left(2 \omega^{*}\right)$ is equal to zero if and only if $\omega^{*} \in\{0, \pi, 2 \pi\}$. For $\omega^{*} \in\{0,2 \pi\}$, the limit $\lim _{\omega \rightarrow \omega^{*}} \lambda^{0}(\omega) / \lambda^{0}(2 \omega)$ exists. In the case $\omega^{*}=\pi$, the function $H(\omega)$ has a zero at least of order $3(N \geq 3)$, see [6] or [7]. However, the zero of $\lambda^{0}(2 \omega)$ in $\omega^{*}=\pi$ is only of order 2, Lemma 2.1 (i) in [16]. Consequently, $E_{1}$ and $E_{2}$ are finite and hence,

$$
\frac{\left|H\left(\omega_{\mu}\right) H\left(\omega_{\nu}\right)\right| \Lambda_{m ; \mu, v}}{\Lambda_{2 m ; 2 \mu, 2 v}} \leq C_{1}, \quad C_{1} \neq C_{1}\left(\varepsilon, \delta_{l}, m\right) .
$$

Next,

$$
\begin{aligned}
\frac{\left|G\left(\omega_{\mu}\right) H\left(\omega_{v}\right)\right| \Lambda_{m ; \mu, v}}{A_{2 m+1 ; 2 \mu, 2 v}} & \leq 4 \sup _{0 \leq \omega, \eta \leq 2 \pi} \frac{|G(\omega) H(\eta)|\left(\varepsilon \lambda^{m}(\omega)+\lambda^{0}(\eta)+\delta_{l}^{2}\right)}{\varepsilon \lambda^{2 m+1}(2 \omega)+\lambda^{0}(2 \eta)+\delta_{l-1}^{2}} \\
& \leq 4 \max \{\underbrace{\left\{\sup _{0 \leq \omega \leq 2 \pi} \frac{\lambda^{m}(\omega)}{\lambda^{2 m+1}(2 \omega)}\right.}_{=E_{3}}, \underbrace{\sup _{0 \leq \eta \leq 2 \pi} \frac{|H(\eta)| \lambda^{0}(\eta)}{\lambda^{0}(2 \eta)}}_{=E_{2}}, \frac{\delta_{l}^{2}}{\delta_{l-1}^{2}}\}
\end{aligned}
$$

Using (A.7) we are able to estimate $E_{3} \leq \max _{\omega} \lambda^{1}(\omega) / \min _{\omega} \lambda^{1}(\omega)$ for $m \geq 1$. Hence, $E_{3}$ is uniformly bounded in $m$ and

$$
\frac{\left|G\left(\omega_{\mu}\right) H\left(\omega_{v}\right)\right| \Lambda_{m ; \mu, v}}{\Lambda_{2 m+1 ; 2 \mu, 2 v}} \leq C_{2}, \quad C_{2} \neq C_{2}\left(\varepsilon, \delta_{l}, m\right) .
$$

Finally, let $j$ be 0 or 1 , then

$$
\begin{aligned}
\frac{\left|\Lambda_{2 m ; 2 \mu, 2 \nu}^{c}\right|}{\Lambda_{2 m+j ; 2 \mu, 2 \nu}} & \leq 4 \sup _{0 \leq \omega, \eta \leq 2 \pi} \frac{\varepsilon\left|\lambda^{m, c}(2 \omega)\right|}{\varepsilon \lambda^{2 m+j}(2 \omega)+\lambda^{0}(2 \eta)+\delta_{l-1}^{2}} \\
& \leq 4 \underbrace{\sup _{0 \leq \omega \leq 2 \pi} \frac{\left|\lambda^{m, c}(2 \omega)\right|}{\lambda^{2 m+j}(2 \omega)}}_{=E_{4}} .
\end{aligned}
$$

We have that $\left|\lambda^{m, c}(2 \omega)\right| \leq \max _{\omega} \lambda^{m}(\omega)$ by (B.1) and by $|H(\omega) H(\omega+\pi)| \leq 1 / 2$. The cases $j=1$ and $j=0$ with $m \geq 1$ yield $E_{4} \leq \max _{\omega} \lambda^{1}(\omega) / \min _{\omega} \lambda^{1}(\omega)$. It remains to consider $j=0$ and $m=0$. However, this was already done in Lemma 2.4 of [16]. Altogether we have shown that

$$
\frac{\left|\Lambda_{2 m: 2 \mu, 2 v}^{c}\right|}{\Lambda_{2 m+j ; 2 \mu, 2 v}} \leq C_{3}, \quad C_{3} \neq C_{3}\left(\varepsilon, \delta_{l}, m\right),
$$


for $j=0,1$. We finish the proof of Lemma B.2 by

$$
\begin{aligned}
& A_{m ; \mu, v}\left|r_{\mu, v}\right| \leq C_{1}\left(1+(1-\sigma)^{-1}\left(\sigma+C_{3}\right)\right), \\
& A_{m ; \mu, v}\left|t_{\mu, v}\right| \leq C_{2}(1-\sigma)^{-1}\left(C_{3}+1\right),
\end{aligned}
$$

where we have used (B.5), (B.6), (B.7) and (B.8).

Proof of Lemma B.1: We will need the estimate $\left\|\left(I-U^{t} U\right) A_{l, m}^{-1}\right\| \leq C^{\prime} \delta_{l}^{2}$ which can be verified by a simple modification of the proof of Lemma 5.3 in [16]. The constant $C^{\prime}$ does not depend on $\varepsilon, \delta_{l}$ or $m$. Now,

$$
\begin{aligned}
\left\|A_{l, m}^{-1}-U^{t}\left(U A_{l, m} U^{t}\right)^{-1} U\right\| & =\left\|\left(I-U^{t}\left(U A_{l, m} U^{t}\right)^{-1} U A_{l, m}\right)\left(I-U^{t} U\right) A_{l, m}^{-1}\right\| \\
& \leq\left\|\left(I-U^{t}\left(U A_{l, m} U^{t}\right)^{-1} U A_{l, m}\right)\right\|\left\|\left(I-U^{t} U\right) A_{l, m}^{-1}\right\| \\
& \leq C_{A} \delta_{l}^{2}
\end{aligned}
$$

with $C_{A}=(1+C) C^{\prime}$ where the constant $C$ is as in Lemma B.2.

\section{References}

[1] Bank, R. E., Dupont, T. F., Yserentant, H.: The hierarchical basis multigrid method. Numer. Math. $52,427-458(1988)$.

[2] Beylkin, G.: On the representation of operators in bases of compactly supported wavelets. SIAM J. Numer. Anal. 6, 1716-1740 (1992).

[3] Bramble, J. H., Pasciak, J. E., Wang, J., Xu, J.: Convergence estimate for multigrid algorithms without regularity assumptions. Math. Comp., 57, 427-458 (1991).

[4] Ciarlet, P. G.: The finite element methods for elliptic problems. New York: North-Holland 1987.

[5] Coifman, R. R., Meyer, Y., Wickerhauser, M. V.: Size properties of wavelet packets. In: Ruskai (ed.) Wavelets and their applications, pp. 453-470. Boston: Jones and Bartlett 1992.

[6] Daubechies, I.: Orthonormal bases of compactly supporte wavelets. Comm. Pure Appl. Math. 41, 906-966 (1988).

[7] Daubechies, I.: Ten lectures on wavelets. CBMS-NSF Series in Applied Mathematics. Philadelphia: SIAM Publications 1992.

[8] Davis, P. J.: Circulant matrices. New York: John Wiley 1979.

[9] Eirola, T.: Sobolev characterization of solutions of dilation equations. SIAM J. Math. Anal. 23, 1015-1030 (1992).

[10] Hackbusch, W.: Multi-grid methods and applications. Springer Series in Computational Mathematics. New York: Springer 1985.

[11] Hackbusch, W.: The frequency decomposition multi-grid method, part I: Application to anisotropic equations. Numer. Math. 56, 229-245 (1989).

[12] Hackbusch, W.: The frequency decomposition multi-grid method, part II: Convergence analysis based on the additive Schwarz method. Numer. Math. 63, 433-453 (1992).

[13] Latto, A., Resnikoff, H. L., Tenenbaum, E.: The evaluation of connection coefficients of compactly supported wavelets. In: Proceedings of the USA-French Workshop on Wavelets and Turbulence. Princeton University 1991.

[14] Mallat, S.: Multiresolution approximation and wavelet orthonormal bases of $L^{2}(\mathbf{R})$. Trans. Amer. Math. Soc. 315, 69-87 (1989).

[15] Meyer, Y:. Wavelets: algorithms and applications. Philadelphia: SIAM Publications 1993.

[16] Rieder, A., Wells, R. O., Jr., Zhou, X.: A wavelet approach to robust multilevel solvers for anisotropic elliptic problems. Technical Report 93-07, Rice University, 1993. Computational Mathematics Laboratory. Accepted for publication in Appl. Comput. Harmonic Anal. (ACHA)

[17] Wang, J.: Convergence analysis without regularity assumptions for multigrid algorithms based on SOR smoothing. SIAM J. Numer. Anal. 29, 987-1001 (1992). 
[18] Wells, R. O., Jr., Zhou, X.: Wavelet interpolation and approximate solutions of elliptic partial differential equations. In: Wilson, R., Tanner, E. A. (eds.) Noncompact lie groups. Dordrecht: Kluwer, 1994. To appear. Proceedings of NATO Advanced Research Workshop.

\section{A. Rieder}

Computational Mathematics Laboratory

Rice University

Houston

TX $77251-1892$

and

Universität des Saarlandes

66041 Saarbrücken

Federal Republic of Germany
X. Zhou

Computational Mathematics Laboratory

Rice University

Houston

TX 77251-1892

U.S.A. 